\title{
Thermodynamic efficiencies of an idealized global climate model
}

\begin{abstract}
We employ the heat engine framework to derive a simple method for assessing the strength of irreversible processes in global climate models (GCMs). Using the explicit energy budget of an idealized GCM, we show that the thermodynamic efficiencies based on the net heating rate and frictional work rate provides a measure of physical and numerical irreversibilities present in either open (e.g., the Hadley circulation) or closed (e.g., the general circulation) circulations. In addition, we show that the Carnot efficiency is useful for assessing the maximum possible efficiency attained by closed circulations. Comparison of the work-based efficiency with that based on the net heating rate and the Carnot efficiency provides a gauge of how close to reversible and ideal the circulations are. A series of experiments with the idealized GCM demonstrate the usefulness of our method and show the sensitivity of an essentially reversible model to changes in physical and numerical parameters such as rotation period and resolution.
\end{abstract}

\section{Introduction}

The earth's atmosphere absorbs energy at higher temperature than the one at which it emits it back to space and, as a result, the atmospheric system is capable of doing work. The work available from the atmospheric heat engine is used to generate convective motions which

D. K. Adams $(\bowtie)$

Scripps Institution of Oceanography Center for Atmospheric Sciences, University of California, San Diego, 9500 Gilman Drive,

Mail Code 0221, La Jolla, CA 92093-0221, USA

E-mail: dadams@fiji.ucsd.edu

N. O. Rennó

Department of Atmospheric, Oceanic and Space Sciences, University of Michigan, Ann Arbor, MI 48109, USA transport energy from heat sources (low-latitude and near-surface regions) to cold sinks (high-latitude and upper-troposphere regions). In steady-state, the kinetic energy generated by the motions resulting from the work done by the atmospheric heat engine is balanced by frictional dissipation. Almost 40 years ago, Lorenz (1967) stated in his classical work on the atmospheric general circulation that the determination and explanation of the thermodynamic efficiency of the global circulation constitutes one of the most fundamental observational and theoretical problems of atmospheric energetics. Unfortunately, how efficiently the general circulation converts heat into the work that maintains atmospheric motions against frictional dissipation still remains an open question in the atmospheric sciences. In this paper, we explore this efficiency question in the context of an idealized climate model.

Convective phenomena varying over a wide range of spatial and temporal scales have been idealized as natural heat engines. Rennó and Ingersoll (1996), Craig (1996), and Emanuel and Bister (1996) have idealized steady-state atmospheric convection as a natural heat engine in order to estimate convective available potential energy (CAPE), convective velocities, and the fractional area covered by convection. Emanuel (1986) has estimated the maximum intensity of hurricanes by treating them as Carnot heat engines. At the smaller end of the spectrum, the heat engine framework provides estimates of the intensity of convective vortices such as dust devils (Rennó et al. 1998), waterspouts (Rennó and Bluestein 2001) and boundary layer convection (Rennó and Ingersoll 1996). In all of the above studies, the atmospheric heat engines were considered to be thermodynamically reversible. However, Rennó (2001) generalized the atmospheric heat engine framework to include all possible irreversible processes and presented evidence that natural convection is, to a first approximation, reversible. In steady-state, work is done on an atmospheric circulation to balance it against frictional dissipation. Hence, the frictional dissipation becomes a measure of 
the work available from the reversible atmospheric heat engine that drives the circulation. Therefore, non-zero work is performed and non-zero thermodynamic efficiencies can be calculated (Goody 2003). In addition, Rennó and Ingersoll (1996) show that a fraction, $\gamma$, of the energy consumed by frictional dissipation returns to the heat engine as part of the heat input while the remaining portion is rejected to the surrounding atmosphere (the heat sink) and ultimately to space (see Fig. 4 of their article). When $\gamma>0$ the thermodynamic efficiency of the convective heat engine is increased.

In a series of recent studies, Pauluis et al. (2000), Pauluis and Held (2002a, b) and Goody (2003) have called into question the idealization of quasi steady-state tropical convection as a thermodynamically reversible heat engine. In the studies of Rennó and Ingersoll (1996), Emanuel and Bister (1996) and Craig (1996), ensembles of moist convective systems are treated as reversible heat engines. Their framework assumes that the primary source of irreversibility in convective systems is the frictional dissipation opposing convective motions. Because, in quasi steady-state, the working rate required to maintain convective motions must equal the rate of frictional dissipation, Rennó and Ingersoll (1996) and Emanuel and Bister (1996) were able to estimate the value of CAPE, convective velocity, and fractional area covered by convective drafts. Pauluis et al. (2000) and Pauluis and Held (2002a, b) suggest that the heat engine framework as proposed by Rennó and Ingersoll (1996) and Emanuel and Bister (1996) greatly overestimates the available work from the convective heat engine. Their modeling studies suggest that irreversibilities associated with falling hydrometers and diffusion of water vapor greatly reduces the available work from a reversible heat engine. Indeed, the results of their numerical simulations suggest that nature is extremely irreversible. Rennó (2001), however, has argued that it may actually be their numerical models that are highly irreversible since predictions from the reversible heat engine framework are close to what is observed on convective circulations in nature.

A number of other studies have also examined irreversibilities and their effects on the entropy budgets of numerical models (e.g., Johnson 1997; Johnson et al. 2000). Johnson (1997) argued that positive definite aphysical sources of entropy in general circulation models can lead to a "general coldness" in their steadystate climates. In this study, we present a simple method which could be used for assessing the amount of irreversibilities in global climate models (GCM) or assimilated data based solely on the explicit energy budget. Treating the model circulations as heat engines, our methodology lies in determining and comparing the values of three thermodynamic efficiencies: a work-based efficiency, a reversible efficiency, and the Carnot efficiency.

The work-based efficiency is simply the ratio of the work performed by the circulation (derived from the kinetic energy budget) to the heat input into its control volume. In steady-state, the work done by a circulation is equal to the rate at which it is consumed by friction opposing it. The reversible efficiency is solely based on the heat budget of the circulation being examined; it is equal to the ratio of the net heating rate to the heat input rate into the circulation's control volume. The Carnot efficiency is the third efficiency calculated and represents the highest efficiency possible for given heat source and sink temperatures. The calculation of these three efficiencies is presented in sect. 3.2. Assessing the strength of irreversibilities comes from comparing the workbased efficiency with the reversible and Carnot efficiencies. For a thermodynamically reversible model, free of numerical or aphysical energy sources or sinks, the rate of net heat input is equal to the rate of working. Thus, the efficiencies based on the kinetic energy budget and the heat budget would be equal to each other. Comparison of these efficiencies provides a measure of the amount of irreversibility associated with parameterization of model physics, numerical diffusion schemes or simply errors inherent in the numerical scheme. The greater the difference between the efficiencies, the greater the amount of irreversibility present in model circulations. For this study, we derive the three different thermodynamic efficiencies for the general circulation -a closed thermodynamic system. In addition, we derive the work-based and reversible thermodynamic efficiencies for an open thermodynamic system and apply it to the Hadley circulation - the zonally symmetric, thermally driven circulation of the tropics. In this case, the circulation exchanges mass and energy with its surroundings. The open-system Carnot efficiency is not derived due to the inherent difficulties in defining the temperatures associated with the net fluxes of energy in and out of the system; i.e., the difficulty in defining heat source and cold sink temperatures for open systems.

Given the uncertainty of how reversible natural and model circulations are, we provide a methodology for evaluating their degree of irreversibility. In the present study, application of this methodology to a highly idealized GCM is an attempt to provide a "proof of concept" application of the framework in the assessment of model irreversibility. That is, this study is not a critique of the validity of this model or its parameterizations and the motivation for the study is to create a general, nonmodel specific thermodynamic framework. We carry out several experiments in which numerical and physical parameters are varied in order to determine how they affect the strength of irreversibilities in the model. These include modification of numerical diffusion (horizontal spectral damping), frictional dissipation, spectral resolution and rotation period.

To begin with, we present a brief overview of the idealized model. The model energy budget is then evaluated in order to quantify the energy not accounted for by explicit physical parameterizations for both the Hadley circulation and global general circulation. This is followed by derivations of the three thermodynamic efficiencies. The results from the series of experiments in 
which the thermodynamic efficiencies are evaluated as a function of changes in model parameters are then presented.

\section{The idealized climate model}

The numerical model employed in this study is the Geophysical Fluid Dynamics Laboratory Idealized GCM (Held and Suarez 1994). This model is highly idealized in its use of simplified physical parameterizations. It consists of an ideal gas atmosphere (dry air) on a rotating sphere with optional topography (not employed in our experiments). The numerical formulation is spectral, semi-implicit, hydrostatic, has hybrid sigmapressure coordinates in the vertical, and employs a leapfrog time-stepping scheme with a time filter to damp the computational mode. A spectral transform method is used in order to evaluate grid-point physical processes and non-linear dynamics. The forcing is particularly simple. It consists of a Newtonian relaxation, whereby the atmospheric temperature field is driven towards a prescribed "radiative equilibrium temperature" which is a function of both latitude and pressure (sigma) level. Dissipation consists of Rayleigh damping of low-level winds which represents boundary layer friction. The specified frictional dissipation of the horizontal winds is linear and is only a function of the pressure (sigma) level. No explicit diffusion is included in the model formulation. Although a scale-selective horizontal numerical mixing is included (damping of highest wavenumber), vertical mixing (diffusion or convection) is not part of the model formulation.

\section{The heat engine framework}

One of the first tasks in the investigation is to calculate the model's energy budget. Accurate assessment of the model's energy budget is critical to the application of the heat engine framework. For the present study, we consider only the model's explicit energy budget. We do not calculate energy losses due to the numerical damping associated with horizontal mixing nor losses due to the numerical scheme (i.e., time marching scheme). Furthermore, energy is not conserved since the frictional dissipation of mechanical energy is not converted into internal energy; it is simply lost. Our calculations, therefore, are based solely on the explicit energy budget of the model and the unaccounted for energy terms are lumped into an "error" term. The use of the word "error" is not meant to imply incorrectness in the model formulation. It includes energy terms that are directly rejected to an external reservoir. For example, the energy dissipated by friction and not converted into internal energy contributes largely to the "error" term. The damping of vorticity, divergence and temperature also contributes to this "error" term. Nevertheless, it is recognized that the selective damping of high frequency waves is physically well-motivated, representing dissipation at unresolved scales. The specific contribution to the energy and entropy budget of each of the terms covered under the "error" term will be presented in a future study.

For the global circulation, in steady state, the energy budget is determined solely by the heat budget and frictional dissipation. In order to properly determine the energy budget for the steady-state, in Hadley circulation, on the other hand, care must be taken to determine the fluxes of energy across the circulation's boundary. We derive, below, the model energy budget which takes a simple form given the forcing utilized in the idealized GCM.

We obtain the energy equation beginning with the vector form of the momentum equations for a rotating fluid parcel under the influence of gravity with a simple parameterization of frictional dissipation,

$\frac{\mathrm{d} \vec{v}}{\mathrm{~d} t}=-R_{f} \vec{v}-\rho^{-1} \nabla p-2 \vec{\Omega} \times \vec{v}+\vec{g}$.

$R_{f}$ is the Rayleigh friction coefficient. The other quantities follow standard meteorological convention. By taking the dot product of $\vec{v}$ and Eq. 1 and using the identity, $\nabla \cdot(p \vec{v})=p \nabla \cdot \vec{v}+\vec{v} \cdot \nabla p$, we have,

$\rho \frac{\mathrm{d}}{\mathrm{d} t}\left(\frac{v^{2}}{2}+g z\right)=-\rho R_{f} v^{2}-\nabla \cdot p \vec{v}+p \nabla \cdot \vec{v}$.

Using the mass conservation equation in flux form; that is,

$\frac{\partial \rho}{\partial t}=-\nabla \cdot(\rho \vec{v})$,

and the fact that for any scalar quantity $s$, we can write

$\rho \frac{\mathrm{d} s}{\mathrm{~d} t}=\frac{\partial}{\partial t}(\rho s)+\nabla \cdot(\rho s \vec{v})$

Eq. 2 can be rewritten as

$$
\begin{aligned}
& \frac{\partial}{\partial t}\left[\rho\left(\frac{v^{2}}{2}+g z\right)\right]+\nabla \cdot\left[\left(\rho \frac{v^{2}}{2}+\rho g z+p\right) \vec{v}\right] \\
& \quad=-\rho R_{f} v^{2}+p \nabla \cdot \vec{v} .
\end{aligned}
$$

Employing the first law of thermodynamics and the mass conservation equation, the last term in Eq. 5 can be rewritten as,

$p \nabla \cdot \vec{v}=\rho \dot{Q}-{ }_{v} \frac{\mathrm{d} T}{\mathrm{~d} t}$,

where $\dot{Q}$ is the total heating rate. This term includes the fraction $\gamma(0 \leq \gamma \leq 1)$ of the energy consumed by friction that is returned to the convective heat engine (see Rennó and Ingersoll 1996). In numerical models that do not include frictional heating such as the one used in this study, $\gamma=0$ and the energy dissipated is considered to be directly rejected to space. Traditionally, global climate 
models have also ignored the frictional heating term. Substituting this into Eq. 5 and using Eq. 4 to rewrite the scalar $c_{v} T$, the energy equation can be rewritten as

$$
\begin{aligned}
& \frac{\partial}{\partial t}\left[\rho\left(\frac{v^{2}}{2}+c_{v} T+g z\right)\right]+\nabla \\
& \quad \cdot\left[\left(\rho \frac{v^{2}}{2}+\rho c_{v} T+\rho g z+p\right) \vec{v}\right] \\
& \quad=\rho \dot{Q}-\rho R_{f} v^{2} .
\end{aligned}
$$

Integrating this equation over the volume, $V$, of the circulation of interest, we have

$$
\begin{gathered}
\iiint \frac{\partial}{\partial t}\left[\rho\left(\frac{v^{2}}{2}+c_{v} T+g z\right)\right] \mathrm{d} V+\iint\left(\rho \frac{v^{2}}{2}+\rho c_{v} T+\rho g z+p\right) \vec{v} \cdot \hat{n} d A \\
=\iiint \rho \dot{Q} \mathrm{~d} V-\iiint \rho R_{f} v^{2} \mathrm{~d} V,
\end{gathered}
$$

where the divergence equation has been used and $\hat{n}$ is the unit normal vector to the area, $A$, which bounds the control volume containing the circulation. This equation can be applied to the Hadley cell with energy fluxes measured along the latitudinal and vertical boundaries of the circulation. For the global integral, the energy flux term simply disappears as there are no energy fluxes across boundaries.

In our assessment of the model energy budget, we consider only steady-state values. This implies that the first term in Eq. 8 is negligible. From this simplification, it can be seen that for the Hadley circulation, the heating and dissipation rates within the cell are balanced by the energy fluxes (potential, internal and kinetic) across, and pressure work rate, on the boundaries. For the global atmosphere in steady-state, the net heating rate simply balances the energy dissipation rate by friction.

A further simplification arises from the fact that the model is hydrostatic. This means that we can rewrite the flux of internal energy and pressure work rate term in Eq. 8 in terms of an enthalpy flux $\left(\rho c_{p} T \vec{v}\right)$; that is,

$$
\begin{aligned}
\int & \int \rho\left(\frac{v^{2}}{2}+c_{p} T+g z\right) \vec{v} \cdot \hat{n} \mathrm{~d} A \\
= & \iiint \rho \dot{Q} \mathrm{~d} V-\iiint \rho R_{f} v^{2} \mathrm{~d} V .
\end{aligned}
$$

\subsection{The energy budget}

In order to properly calculate the thermodynamic efficiencies, it is necessary to calculate the energy budget explicitly. For the steady-state global systems, Eq. 9 becomes

$\iiint_{G} \rho \dot{Q} \mathrm{~d} V=\iiint_{G} f v^{2} \mathrm{~d} V$

where the subscript $G$ indicates integration over the volume of the entire atmosphere. The net heating rate term on the left-hand side of Eq. 10 is balanced by the frictional dissipation rate of kinetic energy. The global error is simply the difference between these two terms and can be written in a short-hand form as,

$\widehat{\operatorname{Err}}=\widehat{Q}_{\text {net }}-\widehat{D}$,

where

$\widehat{Q}_{\text {net }}=\iiint_{G} \rho \dot{Q} \mathrm{~d} V=\iiint_{G} \rho\left(\dot{Q}_{(+)}-\dot{Q}_{(-)}\right) \mathrm{d} V$

is the net heating rate (excluding heating resulting from frictional dissipation) integrated over the volume of the atmosphere and

$\widehat{D}=\iiint_{G} \rho R_{f} v^{2} \mathrm{~d} V$

is the frictional dissipation integrated over the volume of the atmosphere. $Q_{(+)}$represents the heat input or heating and $Q_{(-)}$represents cooling. The heating rate specific to this model is calculated as

$\dot{Q}=-\rho c_{p} k_{T}\left(T-T_{e q}\right)$,

where $\dot{Q}$ is the heating rate calculated for each grid cell volume in the model. $\rho$ is the density, $c_{p}$ is specific heat of dry air at constant pressure, and $k_{T}$ is an inverse damping time scale which depends on sigma level and latitude. $T$ is the model temperature and $T_{e q}$ represents a "radiative equilibrium temperature". The total heating rate is simply $\dot{Q}$ integrated (i.e., summed) over the volume of interest.

Figure 1 is a plot of a 1,000-day, global running mean at T42 resolution of the net heating rate, $\widehat{Q}_{\text {net }}$, versus the frictional dissipation, $\widehat{D}$. The 1,000-day running mean difference (error) between the net heating and frictional dissipation rates is approximately $20 \%$ of the value of the frictional dissipation, or of the rate of work done by the global circulation. The error in the energy budget has a dependency on model horizontal resolution. However, at horizontal resolution of $\mathrm{T} 30$ or greater, the decrease in error with corresponding increases in resolution is small.

In the case of steady-state Hadley cell energetics, a similar approach is taken. However, in this case, the fluxes of energy through the boundaries must be taken into account. From Eq. 9, it is clear that the difference between the net heating and frictional dissipation rates should equal the sum of the energy fluxes through the boundaries plus the pressure work rate term. The energy budget for the Hadley cell, including the error, $\overline{\text { Err, can }}$ then be written in short-hand form as

$\left(\widetilde{Q}_{\text {net }}-\widetilde{D}\right)=\widetilde{E}_{(f)}+\widetilde{\text { Err }}$,

where

$\widetilde{Q}_{\text {net }}=\iiint_{H C} \rho \dot{Q} \mathrm{~d} V$ 
Fig. 1 1,000-day running mean of global net heating rate, $\widehat{Q}_{\text {net }}$, and dissipation, $\widehat{D}$, at T42 resolution. Err is the global error term measured at the end of the 1,000-day running mean

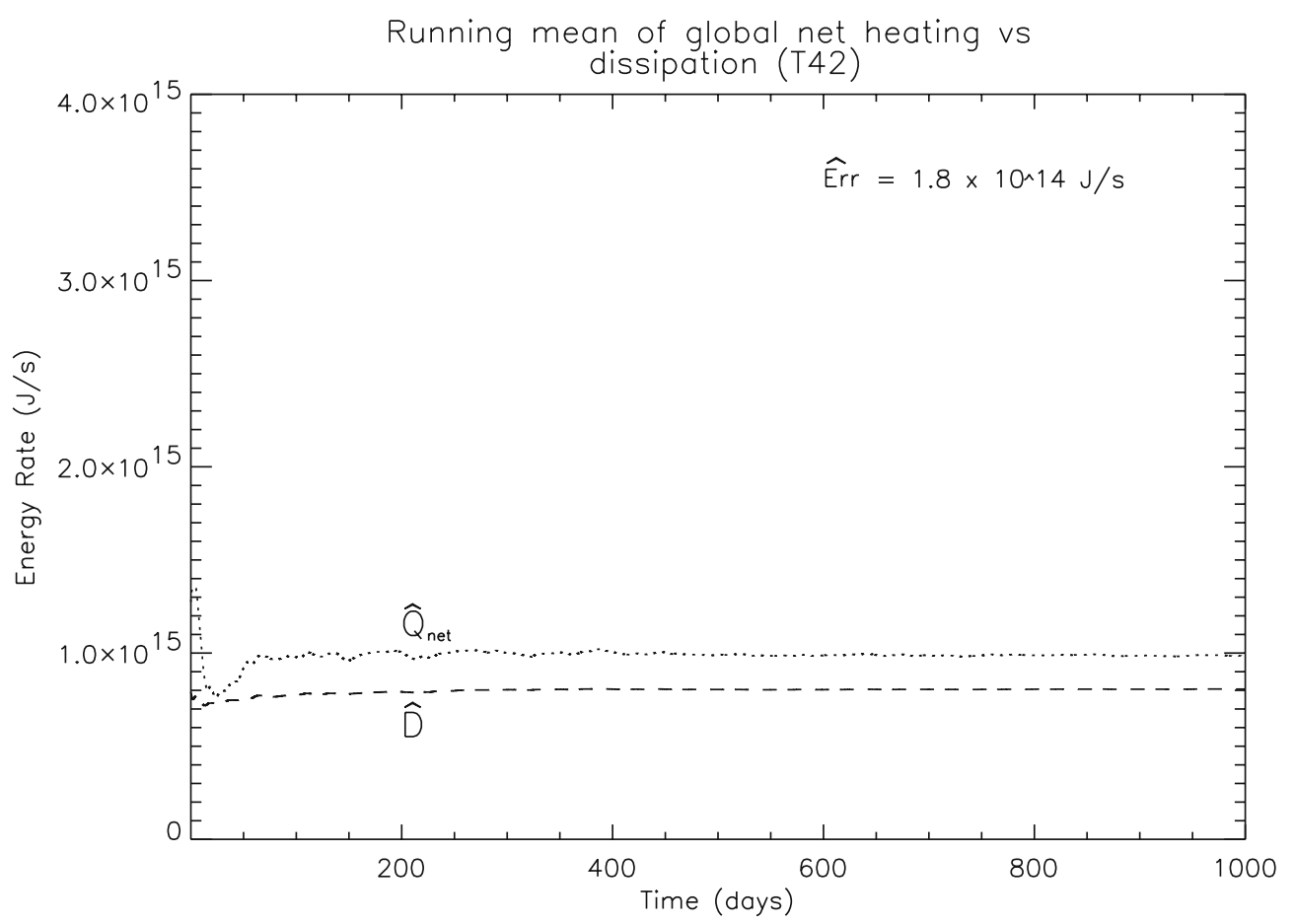

is the net heating rate over the volume of the Hadley cell,

$\widetilde{D}=\iiint_{H C} \rho R_{f} v^{2} \mathrm{~d} V$

is the frictional dissipation within the Hadley cell volume (indicated by the subscript $H C$ ). $\widetilde{E}_{(f)}$ is the lefthand side of Eq. 9, while Err is the error in the Hadley cell energy budget. Figure 2 is a plot of the $\left(\widetilde{Q}_{(\text {net })}-\widetilde{D}\right)$ versus $\widetilde{E}_{(f)}$ for the southern hemisphere Hadley cell with latitudinal boundaries of the control volume placed at $29^{\circ} \mathrm{S}$ and the equator. The Hadley cell error, $\overline{\mathrm{Err}}$, is about $5 \%$ of the value of $\left(\widetilde{Q}_{(\text {net })}-\widetilde{D}\right)$.

\subsection{The Thermodynamic Efficiencies}

By examining the thermodynamic efficiencies of the steady-state circulations, irreversibilities due to changes in model physical parameterizations and numerics can be assessed. In this section, we derive the thermodynamic efficiencies for both open and closed systems. We calculate three thermodynamic efficiencies to gauge the
Fig. 2 1,000-day running mean of energy flux, $\widetilde{E}_{(f)}$, into Hadley cell versus net heating rate, $\widehat{Q}_{\text {net }}$, minus dissipation, $\widehat{D}$, at T42 resolution. Err is the Hadley cell error term measured at the end of the 1,000-day running mean. The latitudinal boundaries for the Hadley cell are at 29 degrees South and the equator

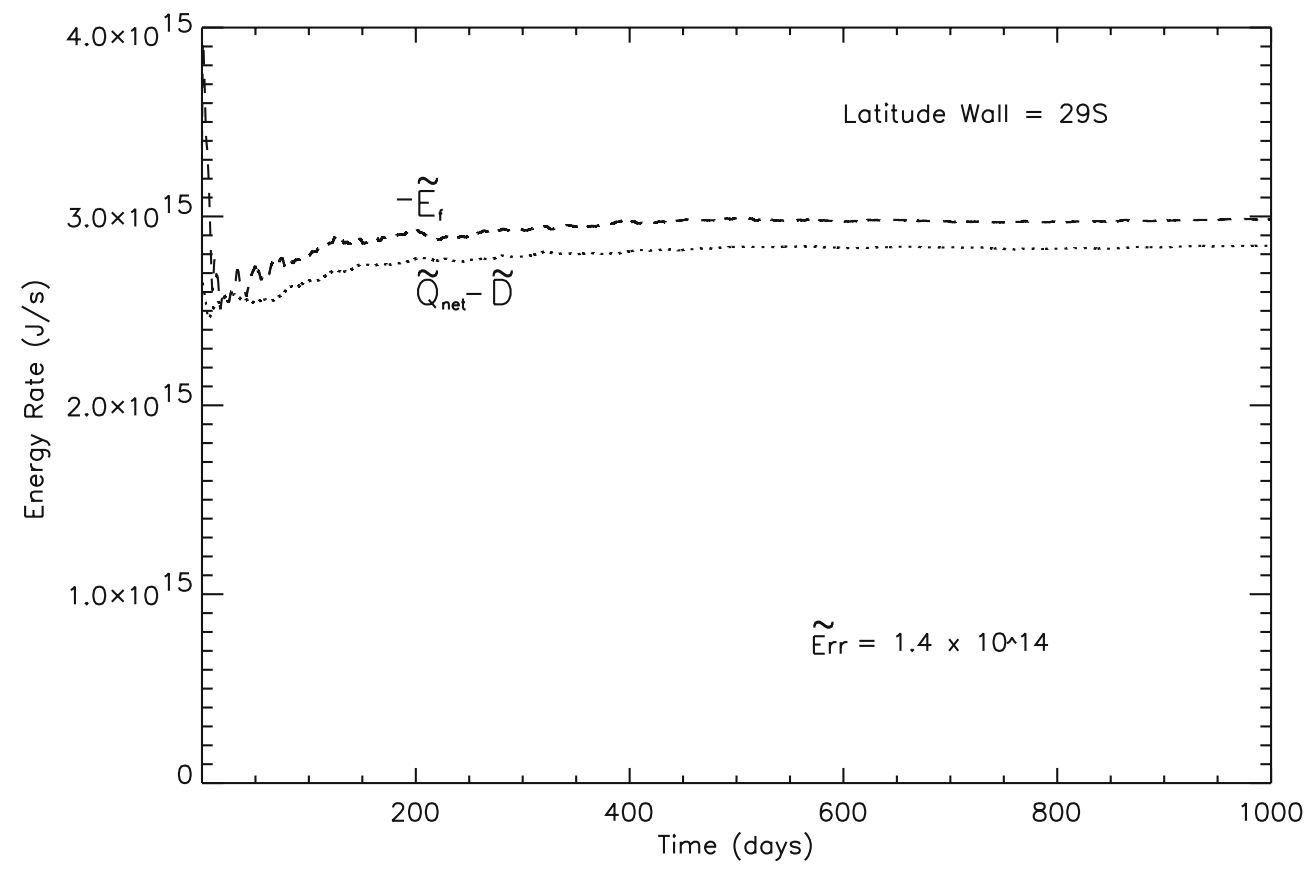


importance of irreversibilities in model-generated circulations. The first method for calculating the efficiency is based on mechanical dissipation of energy (in open systems fluxes of kinetic energy is also necessary to consider); the second is based on the net heating rate, and the third is the Carnot efficiency, or maximum attainable efficiency. In a reversible convective cycle of a system in which the energy budget is correct, the efficiency based on mechanical dissipation and net heating rates would be identical. This is because in the reversible heat engine, the net heat supplied to the engine is converted entirely into work due to the absence of irreversible processes such as heat diffusion and internal friction opposing expansion and compression of the working fluid (see Rennó 2001). The magnitude of the difference between these two efficiencies reflects the importance of irreversibilities in the numerical model.

In order to generalize the heat engine framework to open circulations, we need to decompose the energy budget of a circulation's control volume into work and heating terms.

Equation (9) can be re-written as

$$
\begin{gathered}
\iint \rho\left(\frac{v^{2}}{2}\right) \vec{v} \cdot \hat{n} \mathrm{~d} A+\iiint \rho R_{f} v^{2} \mathrm{~d} V \\
=\iiint \rho \dot{Q} \mathrm{~d} V-\iint \vec{Q}_{f} \cdot \hat{n} \mathrm{~d} A .
\end{gathered}
$$

The sum of the enthalpy and geopotential flux; that is, the dry static energy flux is defined as an "effective" heat flux, $\rho\left(c_{p} T+g z\right) \vec{v} \equiv \vec{Q}_{f}$. Equation 18 shows that the difference between the integrated heating rate and the heat flux through the boundaries of a circulation balances the flux of kinetic energy through the circulation boundaries and the mechanical dissipation of kinetic energy within the circulation. Our approach permits the separation of mechanical energy (1.h.s.) from the heating terms (r.h.s.) for an open heat engine such as the Hadley cell, thereby making it possible to derive the thermodynamic efficiencies based on work and net heating rates.

The thermodynamic efficiency of a reversible heat engine is defined as the fraction of the heat input which is converted to mechanical energy, that is

$$
\eta \equiv \frac{\dot{W}}{\dot{Q}_{+}}
$$

where $\dot{W}$ is the rate of work done by the circulation, $\dot{Q}_{+}$is the heat input rate (see Rennó 2001 for discussion of irreversible heat engine efficiency). In the case of an open system in steady-state, the mechanical energy generated by the heat engine (the bulk fluid motion) is dissipated by friction. However, mechanical energy generated in the control volume may be imported/exported through the boundaries of the volume as kinetic energy fluxes (the first term in Eq. 18). In addition, in order to isolate the work done solely by the heat engine, it is necessary to consider the heat flux across the boundaries of the control volume (the last term in Eq. 18). Using the convention that fluxes into the control volume are negative and outward fluxes are positive, we calculate the thermodynamic efficiency in two ways. In the case where the heat flux is directed into the system; i.e., $\iint \rho\left(\vec{Q}_{f}\right) \cdot \hat{n} \mathrm{~d} A \leq 0$, we have

$\eta=\frac{\iiint \rho R_{f} v^{2} \mathrm{~d} V+\iint \rho\left(\frac{v^{2}}{2}\right) \vec{v} \cdot \hat{n} \mathrm{~d} A}{\iiint \rho \dot{Q}_{(+)} \mathrm{d} V-\iint \rho\left(\vec{Q}_{f}\right) \cdot \hat{n} \mathrm{~d} A}$

because the heat flux through the boundaries of the control volume adds to the volume's heat input. From this calculation, it is apparent that large fluxes of dry static energy into the volume of interest decrease the overall efficiency as do influxes of kinetic energy. In the case in which the fluxes of dry static energy are outward from the system, they are no longer part of the heat input into the heat engine. As a result, the thermodynamic efficiency, $\eta$, is calculated as

$\eta=\frac{\iiint \rho R_{f} v^{2} \mathrm{~d} V+\iint \rho\left(\frac{v^{2}}{2}\right) \vec{v} \cdot \hat{n} \mathrm{~d} A}{\iiint \rho \dot{Q}_{(+)} \mathrm{d} V}$

because the heat flux through the boundaries of the control volume represents a loss of energy from the volume's heat input. In order to calculate the thermodynamic efficiency for the global circulation (a closed system), the only modification is to eliminate the flux terms. The global efficiency based on frictional dissipation of energy is, therefore, calculated as

$\eta=\frac{\iiint \rho R_{f} v^{2} \mathrm{~d} V}{\iiint \rho \dot{Q}_{(+)} \mathrm{d} V}$

The above derivations are based on the working rate; that is, the frictional dissipation of the bulk fluid motion generated by the heat engine. By comparing these workbased efficiencies with the reversible efficiency based solely on the heat budget, we can assess how close model-generated circulations are to reversible. It follows from the above that the thermodynamic efficiency of an open reversible heat engine, based on the net heating rate, is

$\eta_{\mathrm{rev}}=\frac{\iiint \rho \dot{Q}_{(+)} \mathrm{d} V-\iint\left(\rho\left(\vec{Q}_{f}\right) \cdot \hat{n} \mathrm{~d} A-\iiint \rho \dot{Q}_{(-)} \mathrm{d} V\right.}{\iiint \rho \dot{Q}_{(+)} \mathrm{d} V-\iint\left(\rho\left(\vec{Q}_{f}\right) \cdot \hat{n} \mathrm{~d} A\right.}$,

where the same sign convention for fluxes applies. In those cases when the fluxes of dry static energy are directed outward from the open system, the reversible efficiency maybe be written as

$\eta_{\mathrm{rev}}=\frac{\iiint \rho \dot{Q}_{(+)} \mathrm{d} V-\iint\left(\rho\left(\vec{Q}_{f}\right) \cdot \hat{n} \mathrm{~d} A-\iiint \rho \dot{Q}_{(-)} \mathrm{d} V\right.}{\iiint \rho \dot{Q}_{(+)} \mathrm{d} V}$

For the closed system we have, 
$\eta_{\mathrm{rev}}=\frac{\iiint \rho \dot{Q}_{(+)} \mathrm{d} V-\iiint \rho \dot{Q}_{(-)} \mathrm{d} V}{\iiint \rho \dot{Q}_{(+)} \mathrm{d} V}$.

Since $\eta_{\text {rev }} \geq \eta$, the difference between the two numbers provides a measure of just how large irreversibilities are. This is discussed in the following section in the context of the idealized GCM. For this highly simplified GCM, the difference between $\eta_{\text {rev }}$ and $\eta$ is essentially a reflection of energy conservation. However, one could easily imagine a model in which parameterizations of convection, turbulent diffusion or more complex processes are included, whereby the difference between the efficiencies is reflective of irreversibilities due to these parameterizations.

For a final comparison, the Carnot efficiency for the global circulation is calculated. This efficiency is the highest efficiency possible and, therefore, provides an upper bound on the efficiencies of model-generated circulations. It gives a measure of how close to ideal a circulation's heat engine is. In order to properly calculate the Carnot efficiency, it is necessary to weight the temperature of the heat source and sink by the heating and cooling rates, respectively. This gives

$\eta_{c} \equiv \frac{\left[T_{h}\right]-\left[T_{c}\right]}{\left[T_{h}\right]}$,

where $\left[T_{h}\right]$ and $\left[T_{c}\right]$ are calculated in the following manner:

$\left[T_{h}\right]=\frac{1}{\left[\dot{Q}_{(+)}\right]}\left(\iiint \dot{Q}_{(+)} \mathrm{d} V\right)$

where $\left[\dot{Q}_{(+)}\right]$is the input heating integrated over the volume. In the case of $\left[T_{c}\right]$, we have

$\left[T_{c}\right]=\frac{1}{\left[\dot{Q}_{(-)}\right]}\left(\iiint \dot{Q}_{(-)} \mathrm{d} V\right)$

where $\left[\dot{Q}_{(-)}\right]$is the cooling integrated over the control volume. We turn now to applications of these efficiencies to the idealized GCM.

\section{Application of framework and discussion}

In this section, the sensitivity of the various thermodynamic efficiencies to the modification of physical and numerical parameters is studied. These experiments are designed to provide a "proof of concept" of the idea of

Table 1 Global thermodynamic efficiencies versus spectral damping coefficient

\begin{tabular}{lrrrrrr}
\hline Efficiency & \multicolumn{6}{c}{ Spectral damping coefficient $\left(\mathrm{day}^{-1}\right)$} \\
\cline { 2 - 7 } & 0.005 & \multicolumn{1}{c}{0.01} & 0.05 & 0.1 & 0.15 & \multicolumn{1}{c}{0.2} \\
\hline$\eta$ & 9.12 & 9.21 & 8.74 & 8.17 & 6.69 & 7.29 \\
$\eta_{\text {rev }}$ & 12.27 & 12.47 & 11.92 & 12.04 & 11.77 & 12.77 \\
$\eta_{c}$ & 13.24 & 13.49 & 13.34 & 13.49 & 13.64 & 14.64 \\
\hline
\end{tabular}

comparing model efficiencies in the assessment of the importance of irreversibilities. The different experiments can be classified for convenience into two categories: (1) model numerics and (2) model forcing. For the three efficiency calculations, steady-state values and at T30 resolution were employed. We show below that T30 provides sufficient horizontal resolution for these efficiency calculations. Specifically, the experiments were started from an isothermal state at rest with small perturbations added to break the symmetry. Integrations were carried out for 1,000 model days (54,000 timesteps); the model statistics having approached a steadystate (see Figs. 1, 2). The 1,000-day mean, calculated from single daily values, was used to identify the latitudinal boundaries of the Hadley cell. The determination of the latitudinal boundaries was based upon the latitude of maximum vertical velocity and convergence of the meridional wind. The 1,000-day mean was then used to restart the experiment. Model integration was then carried forward for 1,000 more days in order to calculate running averages (from all 54,000 timesteps) of the necessary quantities for the steady-state energy budget.

\subsection{Sensitivity to numerical parameters}

Two experiments were carried out in which numerical parameters were modified. These experiments provide a sensitivity test of the model's steady-state solutions to changes in model numerics. The two experiments involved modification of the spectral damping coefficient and changes in the model's spectral resolution. Results are presented for both the general circulation (a closed system) and the Hadley cell (an open system).

\subsubsection{Spectral damping}

The idealized GCM does not have explicit diffusion and only a scale-selective horizontal mixing is included. The form of this mixing of vorticity, divergence and temperature is a Laplacian raised to the fourth power whose strength is set to that of an e-folding time of 0.1 days for the shortest wave. The purpose of this mixing is to prevent the accumulation of energy at the high frequencies to control non-linear instabilities. The spectral damping coefficient was varied by modifying the e-folding time for the smallest wave from between 0.2 days; that is, stronger damping, to values of 0.005 days; that is, weaker damping. Table 1 summarizes the results of these experiments. Reducing the spectral damping results in a more energetic global circulation. Frictional dissipation increases by about $30 \%$ between the experiments with strong and weak damping and $\eta$, the thermodynamic efficiency based on work, increases. The increase in heat input rate is only approximately $10 \%$, and relative changes in $Q_{\text {net }}$ and $Q_{(+)}$are small. Hence, the heat-based and Carnot 
Fig. 3 A plot of global thermodynamic efficiencies versus spectral resolution
Global Efficiencies versus spectral resolution

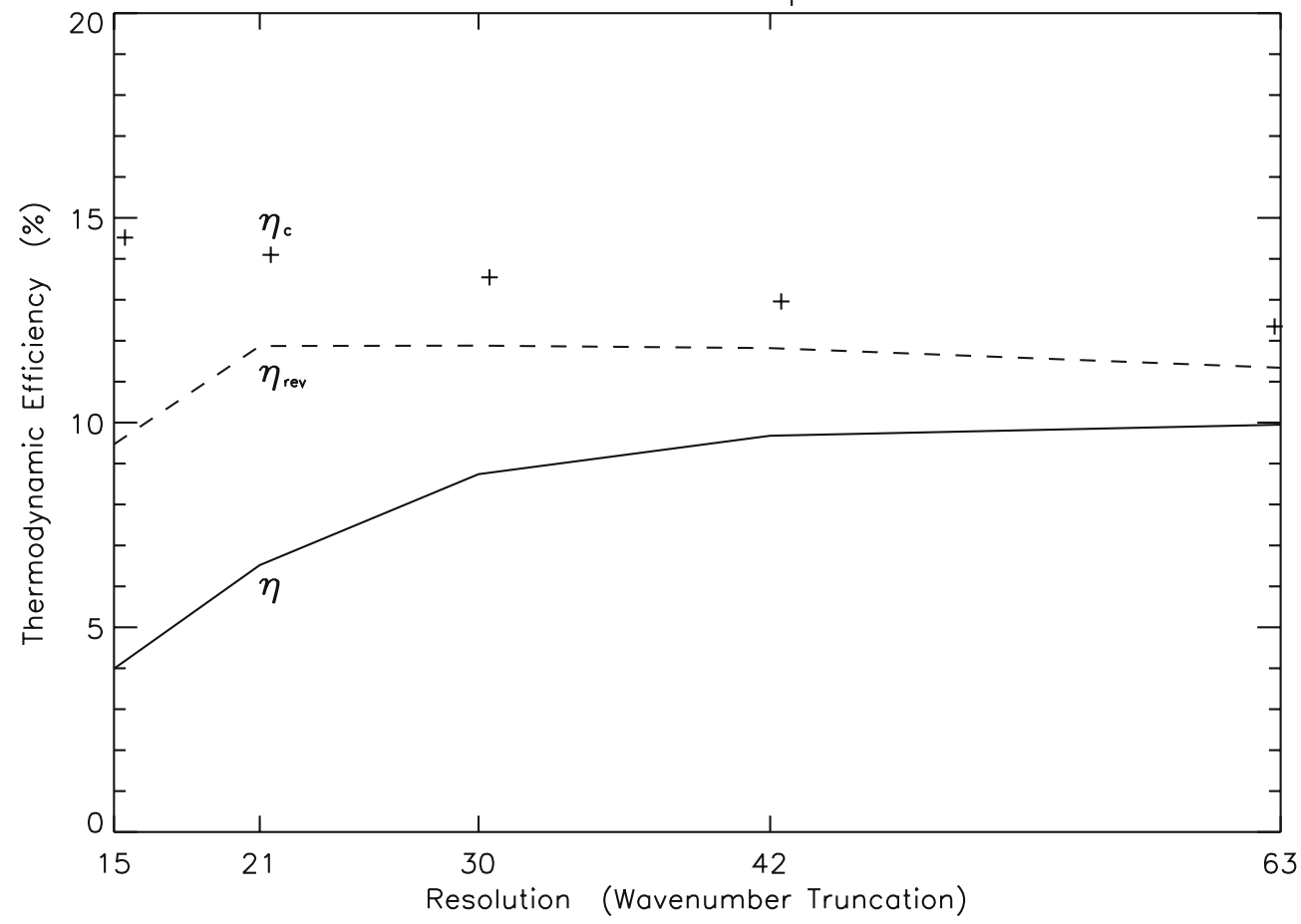

efficiencies, $\eta_{\text {rev }}$ and $\eta_{\mathrm{c}}$, respectively, present relatively small changes implying that the model becomes more irreversible as spectral damping strength increases; that is, as the intensity of horizontal mixing is increased. This can be seen by comparing the three global efficiencies. Thus, increases in the value of the spectral damping makes the model more irreversible. Table 2 summarizes the sensitivity of the Hadley circulation efficiencies to changes in the spectral damping coefficient. Increases in irreversibility of the Hadley circulation as a function of increased spectral damping is not apparent. The relative differences in positive heating and dissipation within the Hadley cell vary little leading to only small differences in the work-based efficiency, $\eta$. Likewise, the relative changes in the heat flux versus net heating rate within the Hadley cell are also small. As a result, there is no obvious trend towards increased irreversibility with strengthened spectral damping. We speculate that this is due to the fact that in the tropical region gradients are weak and most of the energy is in large-scale circulations.

\subsubsection{Spectral resolution}

For climate studies, it is critical to understand model sensitivity to horizontal resolution. Models that demonstrate no tendency to converge towards a single value of the thermodynamic efficiency as resolution is increased would certainly be considered thermodynamically inconsistent. Research by Boer and Denis (1997) suggests that the resolution requirement for properly capturing the dynamical aspects of climate is not that large. Using a simply forced model similar to the model used in our study, they state that T32 is sufficient resolution for the convergence of model dynamics. We have carried out a series of experiments in which the sensitivity of the thermodynamic efficiency is observed as a function of changing horizontal resolution. Our approach is similar to theirs in that we assume known and "correct" physical parameterizations. However, our results focus on the strength of irreversibilities as a function of resolution.

Figure 3 gives the global efficiencies as a function of horizontal resolution. In agreement with Boer and Denis (1997), there appears to be convergence in the workbased and reversible efficiencies at resolution $\geq T 30$. As the resolution is increased, the three efficiencies continue to converge, albeit at a decreased rate. This implies that a simply forced GCM is to a first order, not only reversible, but close to the maximum efficiency possible. The decreasing rate of convergence between the efficiencies at higher resolution also agrees with the results of Boer and Denis (1997) and others who have examined the effects of horizontal resolution on dynamical variables such as eddy fluxes (e.g., Boyle 1993; Boville 1991; Senior 1995).

Table 2 Hadley cell thermodynamic efficiencies versus spectral damping coefficient

\begin{tabular}{lllllll}
\hline Efficiency & \multicolumn{6}{l}{ Spectral damping coefficient $\left(\mathrm{day}^{-1}\right)$} \\
\cline { 2 - 7 } & 0.005 & 0.01 & 0.05 & 0.1 & 0.15 & 0.2 \\
\hline$\eta$ & 5.40 & 5.60 & 5.38 & 4.88 & 4.91 & 6.82 \\
$\eta_{\mathrm{rev}}$ & 8.42 & 8.55 & 8.61 & 7.21 & 8.86 & 8.89 \\
\hline
\end{tabular}


Fig. 4 A plot of Hadley cell thermodynamic efficiencies versus spectral resolution

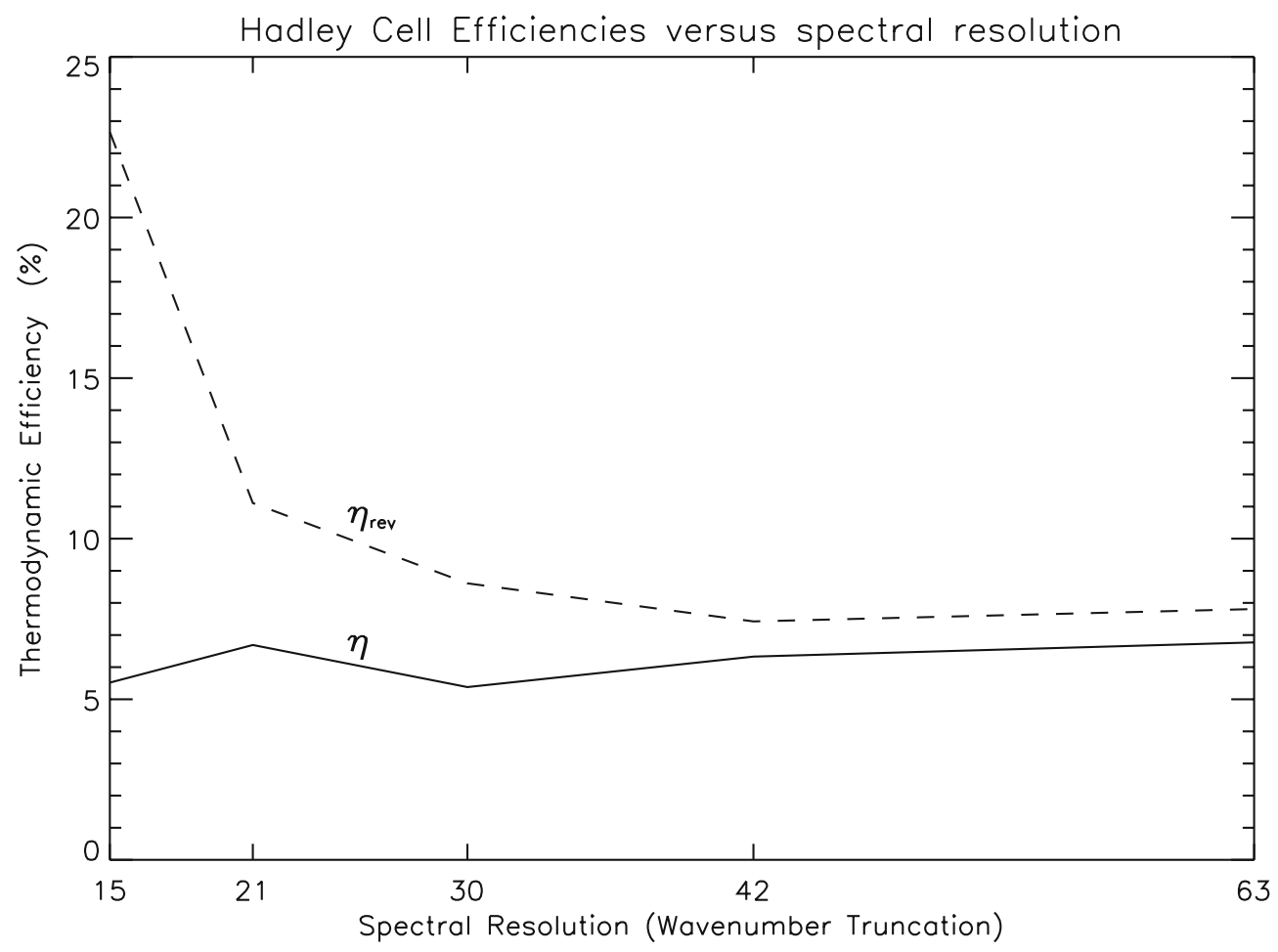

For the Hadley cell, the variation of $\eta$ is small with respect to changes in resolution (Fig. 4). As in the case of spectral damping, the relative differences in positive heating and dissipation within the Hadley cell change little resulting in a near constant $\eta$. $\eta_{\text {rev }}$, on the other hand, varies greatly over the range of spectral resolution. As resolution decreases, there is a relative decrease in the heat flux out of the Hadley cell. Referring to Eq. 24, it can be seen that decreases in outward heat fluxes lead to increase in $\eta_{\text {rev }}$. Our results suggest that model energetics are sensitive to resolution, with coarser resolution leading to greater irreversibility.

\subsection{Sensitivity to model forcing}

\subsubsection{Frictional Damping}

The form of frictional dissipation in the model is a simple linear damping of the velocities, which is solely a
Fig. 5 A plot of global thermodynamic efficiencies versus planetary rotation period

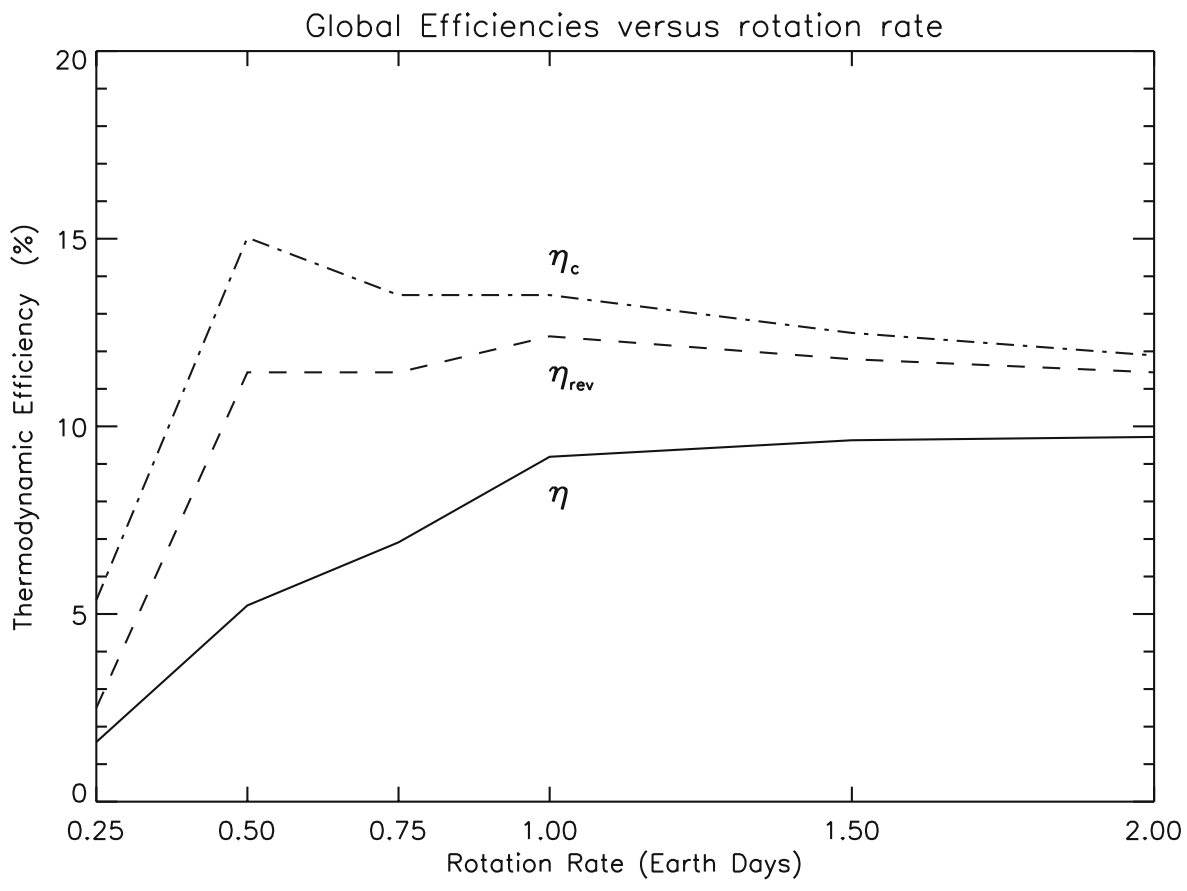


Table 3 Global thermodynamic efficiencies versus frictional damping coefficient

\begin{tabular}{lrrrrrr}
\hline Efficiency & \multicolumn{6}{l}{ Frictional damping coefficient $\left(\mathrm{day}^{-1}\right)$} \\
\cline { 2 - 7 } & \multicolumn{1}{c}{0.1} & \multicolumn{1}{c}{0.2} & \multicolumn{1}{c}{0.5} & 0.67 & \multicolumn{1}{c}{1} & \multicolumn{1}{c}{2} \\
\hline$\eta$ & 8.01 & 9.03 & 9.09 & 8.91 & 8.74 & 8.72 \\
$\eta_{\text {rev }}$ & 12.36 & 12.74 & 12.78 & 12.44 & 11.92 & 11.86 \\
$\eta_{c}$ & 14.53 & 14.13 & 13.95 & 13.56 & 13.34 & 13.03 \\
\hline
\end{tabular}

function of pressure (see Held and Suarez 1994). Several experiments were conducted in which the value of the frictional damping coefficient is varied. The standard value for the frictional damping time is given as 1 day $^{-1}$. This value was varied from 0.1 to $2 \mathrm{day}^{-1}$. Table 3 summarizes the results of these experiments. Global efficiencies are relatively insensitive to changes in the frictional damping coefficient, showing only a slight tendency toward decreased irreversibility with increases in the frictional damping coefficient; i.e., stronger damping. We speculate that this occurs because the explicit friction reduces the intensity of the circulations and make them less diffusive and more reversible. For the Hadley circulation, similar, though somewhat more pronounced behavior is observed (Table 4). With weaker damping, the discrepancy between the workbased and heating-based efficiencies is greater than with stronger damping and, hence, the circulation is more irreversible.

\subsubsection{Rotation period}

The effects of changing the planetary rotation period in the context of numerical models has been the subject of several studies which focused on understanding different general circulation regimes (Hunt 1979; Del Genio and Suozzo 1987; Navarra and Boccaletti 2002). It has been noted that changes in rotation period affect the thermodynamic efficiency of the of the general circulation (Hunt 1979). For this study, the planet's rotation period was modified in several experiments by varying the rotation period between 2 Earth days on the slow end and $1 / 4$ Earth days on the fast end. Figure 5 shows the changes in global efficiences with rotation period. The work-based efficiency, $\eta$, increases from rapid rotation to slower rotation. This variation is consistent with the theoretical studies that show that rotation stabilizes the atmosphere inhibiting convective circulations (Kuo 1956; Hunt 1979). Figure 6 shows, respectively, the streamfunction at $6,12,24$ and $48 \mathrm{~h}$ rotation periods of

Table 4 Hadley cell thermodynamic efficiencies versus frictional damping coefficient

\begin{tabular}{lrrrrrr}
\hline Efficiency & \multicolumn{6}{c}{ Frictional damping coefficient $\left(\mathrm{day}^{-1}\right)$} \\
\cline { 2 - 7 } & \multicolumn{1}{c}{0.1} & \multicolumn{1}{c}{0.2} & 0.5 & 0.67 & \multicolumn{1}{l}{1} & 2 \\
\hline$\eta$ & 5.65 & 5.91 & 6.34 & 5.51 & 5.38 & 4.10 \\
$\eta_{\text {rev }}$ & 13.14 & 10.21 & 9.78 & 8.84 & 8.61 & 6.80 \\
\hline
\end{tabular}

the zonally-averaged meridional circulation. Angular momentum conservation produces larger zonal velocity gradients and reduces the latitudinal extension of the meridional circulations (the Hadley and Ferrel cells) as the rotation rate is increased. The smaller meridional circulations observed at short rotation periods are more turbulent and therefore produce stronger eddy and numerical diffusion values that the circulations with longer rotation periods. The larger values of diffusion at high rotation period leads to circulations of smaller work-based thermodynamic efficiency, $\eta$.

Figure 7 shows that the experiments with shorter rotation periods produces weaker heating and cooling rates that those with longer rotation periods. Moreover, increases in the rotation period shifts the heating rate to the lower altitudes in the equatorial troposphere and the cooling rate to higher altitudes in the polar troposphere. These changes alone would lead to increases in all thermodynamic efficiences. However, changes in $\eta_{\mathrm{rev}}$ and $\eta_{\mathrm{c}}$ with rotation period are more complex than the changes in $\eta$. The reason for the more complex behavior is that these efficiencies are affected not only by changes in the values and distribution of the heating and cooling rates, but also by changes in the intensity of numerical diffusion and errors in the model's energy budget. These processes, in turn, are strongly affected by rotation. The values of $\eta_{\mathrm{c}}, \eta_{\text {rev }}$ and $\eta$ approach each other at the shortest rotation period ( $6 \mathrm{~h}$ day), present maximum difference in values (greatest irreversibility) for the $12 \mathrm{~h}$ day, and again approach each other at longer rotation periods. This unexpected behavior for rotation around $12 \mathrm{~h}$ is partially caused by changes in the heating rate and in errors in the model's energy budget with rotation. The model error is a maximum for rotation period equal to $12 \mathrm{~h}$. We believe that this happens because with this rotation period intense and unsteady meridional circulations of modest latitudinal extent are produced. The circulation obtained with rotation period equal to $6 \mathrm{~h}$ are of smaller latitudinal extent, but much weaker than that obtained with rotation period equal to $12 \mathrm{~h}$. The velocity gradients are smaller and the circulations more steady for rotation periods larger than $12 \mathrm{~h}$.

We do not study variations of the efficiencies for the Hadley circulation with rotation because at high rotation period the latitudinal boundaries of the Hadley cell are highly unsteady making the definition of the cell boundaries difficult. This causes problems for calculation of the energy budget of the circulation and, therefore, of its thermodynamic efficiency.

\section{Concluding remarks}

A simple framework for gauging the importance of irreversibilities in large-scale hydrostatic models has been presented. By properly defining the work and heat input terms, the heat engine framework permits the calculation of the thermodynamic efficiencies of both closed and open circulations. By comparing thermodynamic 


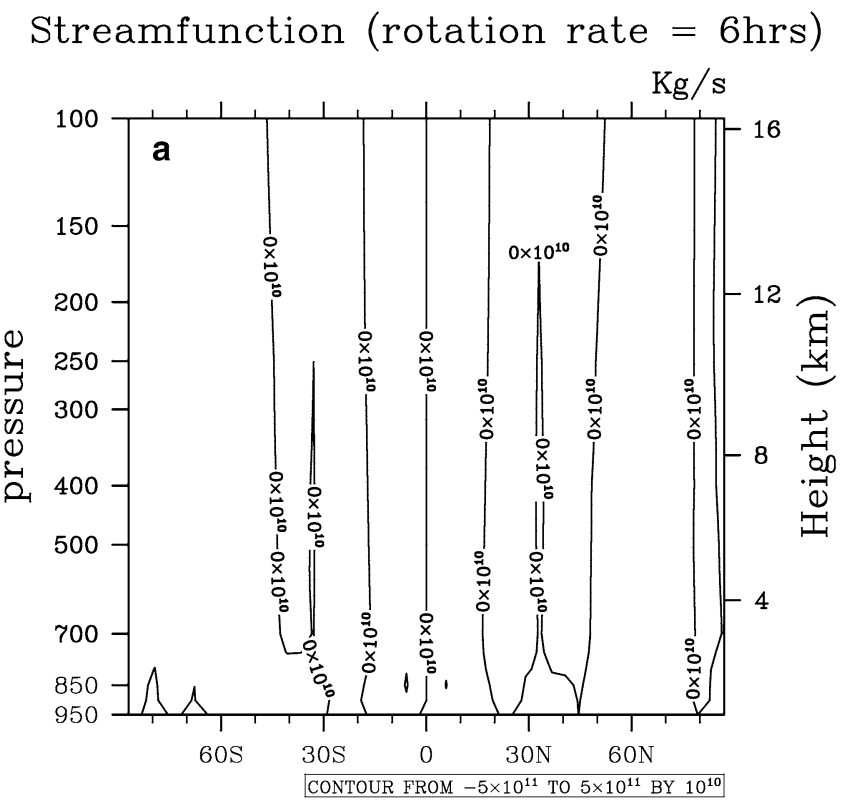

6 hour rotation period

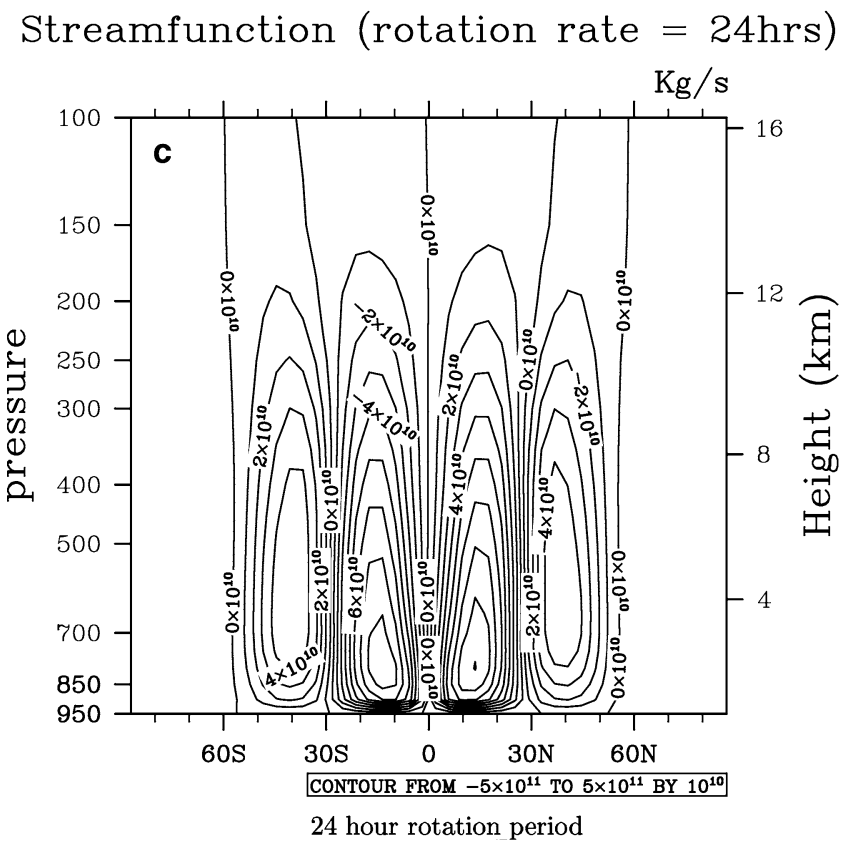

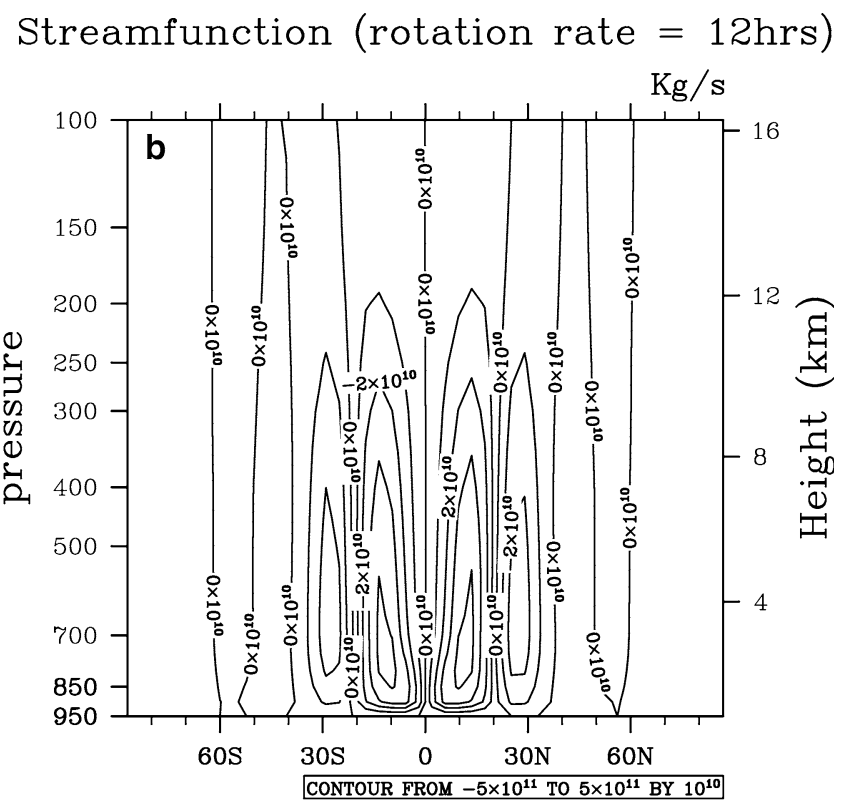

12 hour rotation period
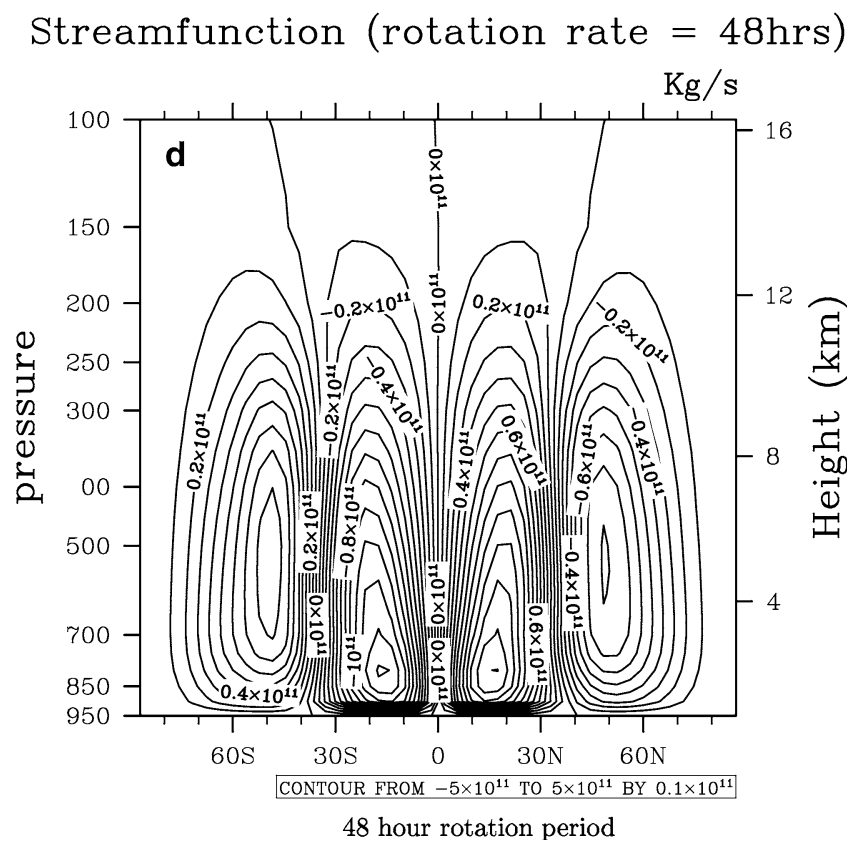

Fig. 6 Plot of the zonally averaged meridional streamfunction, a 6 h rotation period, b 12 h rotation period, c $24 \mathrm{~h}$ rotation period, $\mathbf{d} 48 \mathrm{~h}$ rotation period

efficiencies based on work, heat budget, and the Carnot efficiency, one can gauge the sensitivity that numerical models have with respect to changes in physical and numerical parameters. Our application of this method to a dry, simply forced GCM demonstrates that model irreversibility are sensitive to the values of numerical and physical parameters, such as spectral damping, spectral resolution, and rotation period, among others. Thermodynamically consistent models are expected to produce thermodynamic efficiencies that converge towards specific values as the model resolution is increased. In this case, the thermodynamic efficiencies can be used to determined optimum model resolutions for climate studies. In future work, we intend to apply this framework to a moist model, in order to ascertain the importance of irreversible entropy sources associated with the water substance. In addition, this framework could be applied to assimilated data in order to compare the thermodynamic effiencies of models directly with that of nature. 


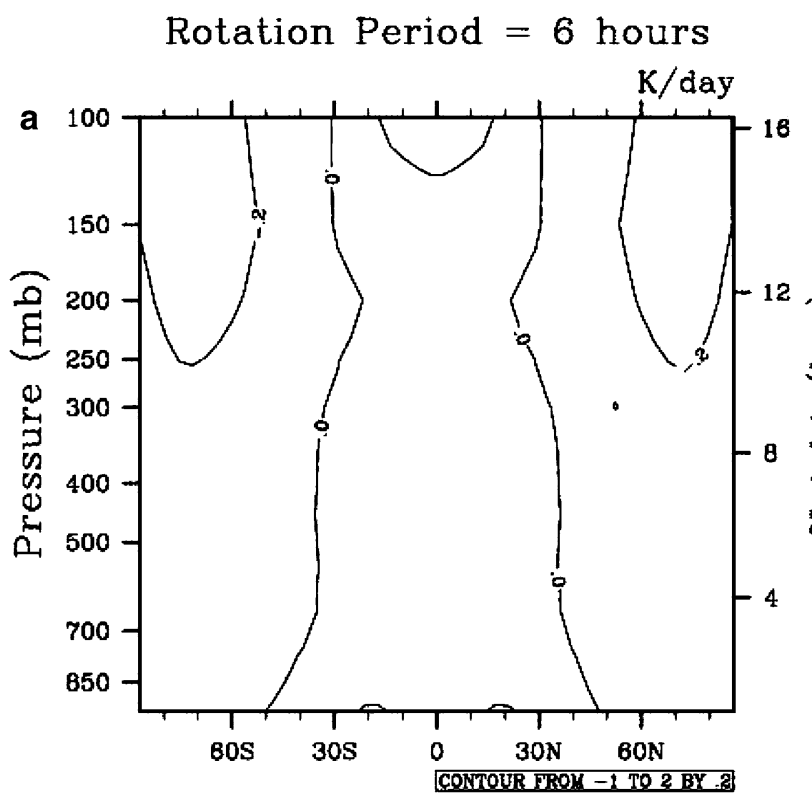

6 hour rotation period

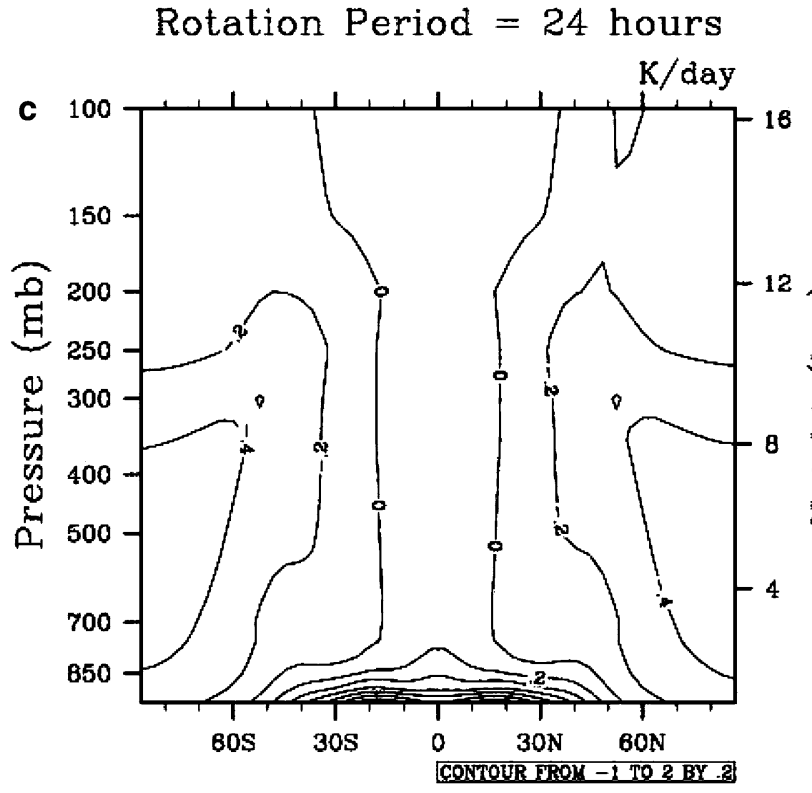

24 hour rotation period
Rotation Period $=12$ hours

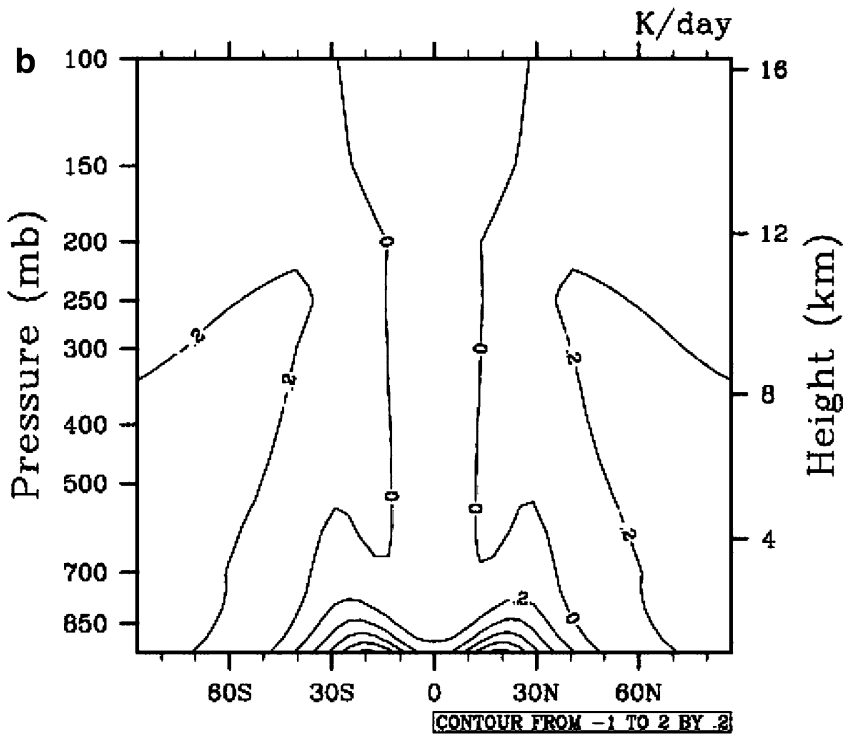

12 hour rotation period
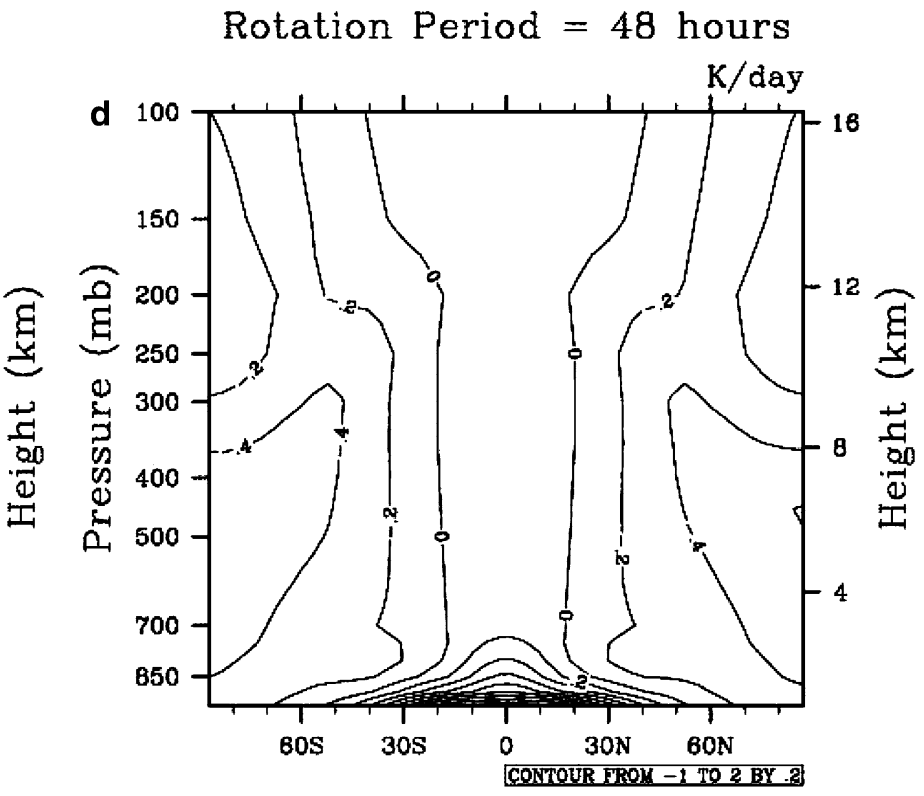

48 hour rotation period

Fig. 7 Plot of the zonally averaged heating rate, a $6 \mathrm{~h}$ rotation period, b $12 \mathrm{~h}$ rotation period, $\mathbf{c} 24 \mathrm{~h}$ rotation period, d $48 \mathrm{~h}$ rotation period

Acknowledgments This work is derived from the first authors' dissertation which was submitted to the University of Arizona. The authors would like to thank The University of Arizona's Department of Atmospheric Sciences and the NSF for supporting this research under grant \#9814120.

\section{References}

Boer GJ, Denis B (1997) Numerical convergence of the dynamics of a GCM. Clim Dyn 13:359-374

Boville BA (1991) Sensitivity of simulated climate to model resolution. J Clim 4:469-485
Boyle JS (1993) Sensitivity of dynamical quantities to horizontal resolution for a climate simulation using the ECMWF (cycle 33) model. J Clim 6:796-815

Craig GC (1996) Dimensional analysis of a convecting atmosphere in equilibrium with external forcing. Q J R Meteorol Soc 122:1963-1967

Del Genio AD, Suozzo R (1987) A comparative study of rapidly and slowly rotating dynamical regimes in a terrestrial general circulation model. J Atmos Sci 44:973-986

Emanuel KA, Bister M (1996) Moist convective velocity and buoyancy scales. J Atmos Sci 53:3276-3285

Emanuel, KA (1986) An air-sea interaction theory for tropical cyclones. Part I: Steady-state maintenance. J Atmos Sci 43:585604 
Goody R (2003) On the mechanical efficiency of deep, tropical convection. J Atmos Sci 60:2827-2832

Held IM, Suarez MJ (1994) A proposal for the intercomparison of the dynamical cores of atmospheric general circulation models. Bull Amer Meteorol Soc 75:1825-1830

Hunt BG (1979) The influence of the earth's rotation rate on the general circulation of the atmosphere. J Atmos Sci 36:13921408

Johnson DR (1997) General coldness of climate models and the second law: implications for modeling the Earth system. J Clim 10:2826-2846

Johnson DR, Lenzen AJ, Zapotocny TH, Schaack TK (2000) Numerical uncertainties in the simulation of reversible isentropic processes and entropy conservation. J Clim 13:38603884

Kuo HL (1956) Energy-releasing processes and stability of thermally driven motions in a rotating fluid. J Meteor 13:82101

Lorenz EN (1967) The nature and theory of the general circulation of the atmosphere. World Meteorological Organization, Geneva, p 161
Navarra A, Boccaletti G (2002) Numerical general circulation experiments of sensitivity to earth rotation rate. Clim Dyn 19:467-483

Pauluis O, Held IM (2002a) Entropy budget of an atmosphere in radiative-convective equilibrium Part I: Maximum work and frictional dissipation. J Atmos Sci 59:125-139

Pauluis O, Held IM (2002b) Entropy budget of an atmosphere in radiative-convective equilibrium Part II: Latent heat transport and moist processes. J Atmos Sci 59:140-149

Pauluis O, Balaji V, Held IM (2000) Frictional dissipation in a precipitating atmospheres. J Atmos Sci 57:989-994

Rennó NO (2001) Comments on Frictional Dissipation in a Precipitating Atmosphere. J Atmos Sci 58:1173-1177

Rennó NO, Bluestein HB (2001) A simple thermodynamic theory for waterspouts. J Atmos Sci 58:927-932

Rennó NO, Ingersoll AP (1996) Natural convection as a heat engine: a theory for CAPE. J Atmos Sci 53:572-585

Rennó NO, Burkett ML, Larkin MP (1998) A simple thermodynamic theory for dust devils. J Atmos Sci 55:3244-3252

Senior CA (1995) The dependence of climate sensitivity on the horizontal resolution of a GCM. J Clim 8:2860-2880 